\title{
Variação temporal na abundância de espécies de aves aquáticas em uma lagoa costeira do Norte Fluminense, sudeste do Brasil
}

\author{
Davi Castro Tavares ${ }^{1,2 *}$ \\ Salvatore Siciliano ${ }^{2}$ \\ ${ }^{1}$ Universidade Estadual do Norte Fluminense Darcy Ribeiro \\ Avenida Alberto Lamego, 2000, CEP 28013-602, Campos dos Goytacazes $\square$ RJ, Brasil \\ ${ }^{2}$ Escola Nacional de Saúde Pública $\square$ FIOCRUZ \\ Rua Leopoldo Bulhões, 1480, sala 611, Manguinhos, CEP 21041-210, Rio de Janeiro $\square$ RJ, Brasil \\ * Autor para correspondência \\ wetlandbirdsbrazil@gmail.com
}

Submetido em 02/06/2013

Aceito para publicação em 09/11/2013

\section{Resumo}

Este estudo avaliou a influência de diferentes regimes de precipitação em flutuações numéricas de aves aquáticas na Lagoa da Ribeira, Quissamã, norte fluminense, Brasil. Contagens de aves foram conduzidas mensalmente entre agosto de 2008 e julho de 2009, totalizando 12 visitas. Dados de precipitação pluviométrica foram obtidos na estação meteorológica automática mais próxima. Um total de 50 espécies foi classificado em seis grupos funcionais. A riqueza e abundância de aves foram maiores durante o período seco (maio/agosto). $\mathrm{O}$ coeficiente linear de Spearman entre a abundância de aves e a precipitação mensal foi -0,77 $(\mathrm{P}=0,003)$. Da mesma forma, houve correlação negativa significativa entre valores de precipitação e a abundância de grupos de aves vasculhadoras, mergulhadoras e catadoras. O modelo de predição exponencial foi o mais adequado aos dados $\left(\mathrm{R}^{2}=0,53\right)$, com coeficiente de correlação entre valores de abundância previstos e os observados, igual a $0,76(\mathrm{P}=0,006)$. O modelo obtido prediz considerável declínio no número total de aves em meses com precipitação superior a $100 \mathrm{~mm}$ na Lagoa da Ribeira.

Palavras-chave: Abundância de aves; Lagoa da Ribeira; Precipitação pluviométrica

\section{Abstract}

Temporal variation in the abundance of waterbird species in a coastal lagoon in the northern Rio de Janeiro, southeastern Brazil. This study evaluated the influence of different rainfall regimes on numerical fluctuations of waterbird species in Ribeira Lagoon, Quissamã, northern Rio de Janeiro state, Brazil. Bird counts were conducted on a monthly basis between August 2008 and July 2009, totaling 12 visits. Rainfall data were obtained at the nearest meteorological automatic station. A total of 50 species were classified into 6 functional groups. Bird richness and abundance were greater during the dry season (May/August). Spearman's coefficient between bird abundance and monthly rainfall was $-0.77(\mathrm{P}=0.003)$. Similarly, there was a significant negative correlation between rainfall values and the abundance of dabbling, diving, and gleaning bird groups. The exponential prediction model was the most appropriate to the dataset $\left(\mathrm{R}^{2}=0.53\right)$, with a correlation coefficient between predicted and observed abundance values of $0.76(\mathrm{P}=0.006)$. The model obtained predicts a significant decline in the total number of birds in months with rainfall over $100 \mathrm{~mm}$ in Ribeira Lagoon.

Key words: Bird abundance; Rainfall; Ribeira Lagoon 


\section{Introdução}

A “Avaliação Ecossistêmica do Milênio" concluiu que a destruição das áreas úmidas tem ocorrido de forma acelerada quando comparada a de outros ecossistemas (WORLD RESOURCES INSTITUTE, 2005). Adicionalmente, a Convenção Ramsar tem reconhecido áreas úmidas com importância internacional e estabelecido metas para sua restauração e preservação (RAMSAR CONVENTION, 2009). Nesse contexto, monitoramentos de aves adquirem especial importância, devido às características ecológicas que tornam esses animais componentes da biodiversidade muito representativos e potenciais indicadores do estado de conservação de ambientes aquáticos (RUTSCHKE, 1987; BLANCO, 1999; VOOREN; BRUSQUE, 1999). De fato, as aves aquáticas sofrem os efeitos imediatos da alteração dos ciclos hidrológicos naturais (ESTEVES, 1998; RODRIGUES; MICHELIN, 2005). Os diferentes regimes de precipitação influenciam diretamente as variáveis físico-químicas da água, sua produtividade, e consequentemente a abundância de alimento e qualidade do habitat disponível para as espécies (MILLAN-NUÑEZ et al., 1982; NESSIMIAN, 1995; HOLLANDA-CARVALHO et al., 2003; MA et al., 2010; MAROTTA et al., 2010). Por isso é essencial compreender como variáveis climáticas influenciam as flutuações temporais de riqueza e abundância aves aquáticas, e consequentemente, a seleção de medidas de manejo e recuperação de áreas úmidas degradadas (ISOLA et al., 2000; TAFT et al., 2002; BOLDUC; AFTON, 2004).

Diferentes autores têm constatado flutuações numéricas de aves aquáticas em resposta à variação da profundidade de corpos d'água e sua influência na disponibilidade de habitat (DIMALEXIS et al., 1997; NTIAMOA-BAIDU et al., 1998; ISOLA et al., 2000). Porém, esse tipo de estudo é escasso no Brasil, onde grande parte das publicações sobre aves aquáticas é focada apenas na descrição de listas de composição. No cenário nacional, o norte do estado do Rio de Janeiro se destaca por possuir extensas áreas úmidas e a segunda maior lagoa do país, a Lagoa Feia (LAMEGO, 1945;
KJERFVE, 1986). Além disso, estudos preliminares relataram elevada abundância de aves aquáticas na região (TAVARES et al., 2012; TAVARES; SICILIANO, 2013b).

A Lagoa da Ribeira, localizada no município de Quissamã, Norte Fluminense, foi reconhecida como Área de Proteção Ambiental em 2006, porém até hoje não dispõe de um plano de manejo. Além disso, sua profundidade e extensão são quase exclusivamente reguladas por regimes de precipitação, o que torna essa lagoa adequada para pesquisas a cerca da influência dos mesmos nas flutuações de abundância de aves aquáticas.

Diante do exposto, o presente estudo tem como objetivos: (1) testar a hipótese de que existe diferença significativa na riqueza e abundância de aves aquáticas entre as estações seca e chuvosa na Lagoa da Ribeira; (2) Correlacionar valores mensais de abundância e precipitação pluviométrica; (3) Ajustar um modelo de predição simples para abundância de aves aquáticas em função de regimes de precipitação.

\section{Material e Métodos}

O trabalho foi desenvolvido na Área de Proteção Ambiental (APA) da Lagoa da Ribeira (22007'12”S, 41 $27^{\prime} 03$ 'O), localizada no município de Quissamã, Norte Fluminense (Figura 1). A área constitui-se de uma zona úmida permanentemente alagada com aproximadamente $5 \mathrm{~km}^{2}$, circundada por charcos, planícies inundáveis e restinga que podem totalizar uma área de até $30 \mathrm{~km}^{2}$, em períodos chuvosos. A lagoa localiza-se a uma distância de aproximadamente $8 \mathrm{~km}$ do mar, e se comunica com a Lagoa Feia, a segunda maior lagoa de água doce do Brasil, por meio de um canal regulado por comportas que permaneceram fechadas ao longo de todo o período de estudo. Dessa maneira, a profundidade da lâmina d'água da lagoa permaneceu regulada basicamente pelos regimes de precipitação. Em adição, a APA da Ribeira situa-se a $2 \mathrm{~km}$ do limite norte do Parque Nacional da Restinga de Jurubatiba. 
FIGURA 1: Área de estudo, com indicação da Lagoa da Ribeira, Quissamã, Norte Fluminense, sudeste do Brasil.

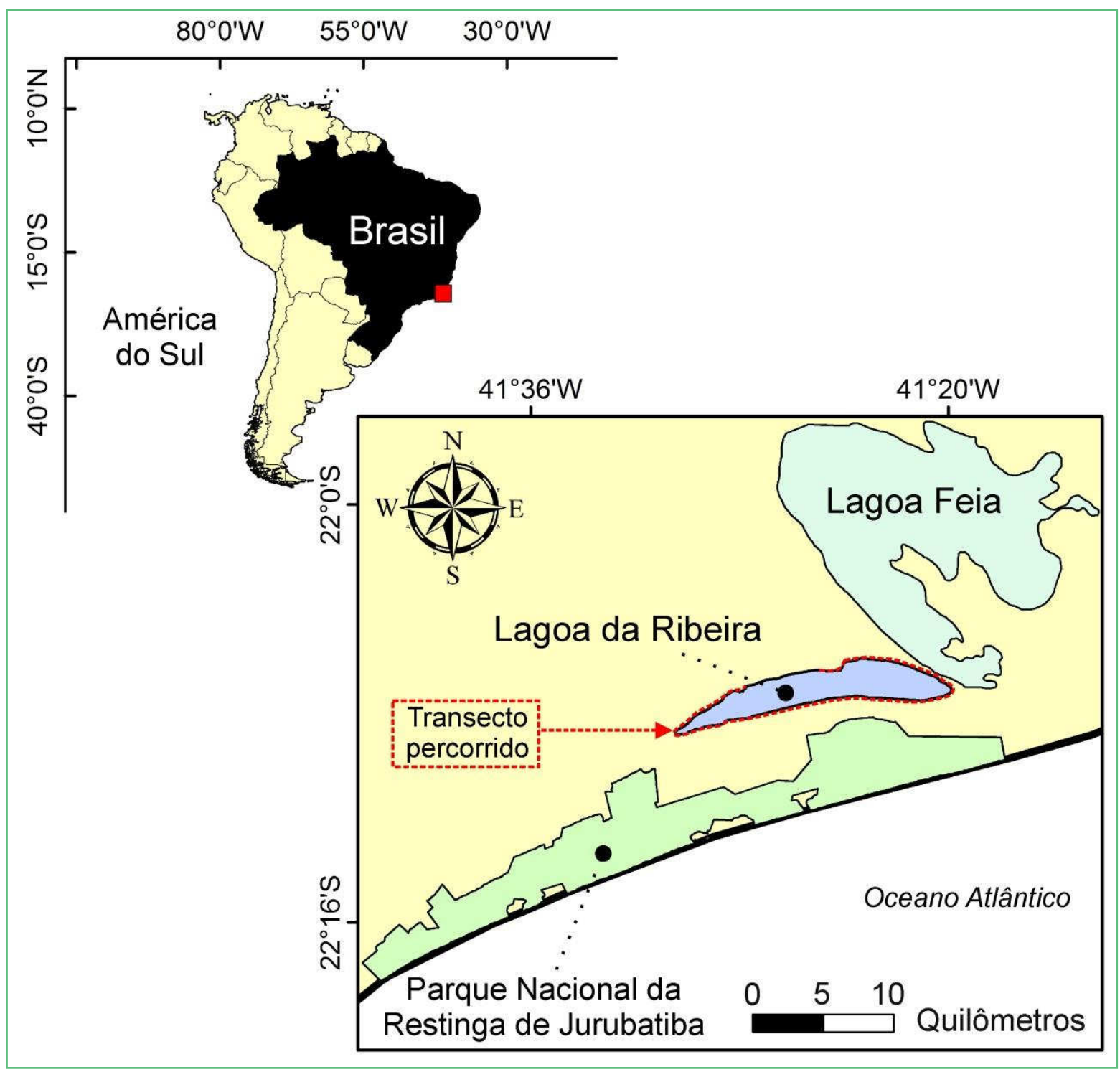

O clima predominante na região é o semi-úmido. As médias de temperatura variam ao longo do ano entre $20,9^{\circ} \mathrm{C}$ em julho e $26,6^{\circ} \mathrm{C}$ em fevereiro, enquanto a média pluviométrica é de $963 \mathrm{~mm}$ anuais (ARAÚJO et al., 1998). Há apenas duas estações marcantes, a seca (de maio a agosto) e a chuvosa (de setembro a abril). Contudo, no período de estudo, a estação úmida ocorreu de novembro a março, e a seca entre abril e outubro.

Excursões mensais à Lagoa da Ribeira foram conduzidas entre os meses agosto de 2008 e julho de 2009, totalizando 12 visitas e 108 horas de observação, preferencialmente entre 6 e $11 \mathrm{~h}$, bem como 15 e $19 \mathrm{~h}$.
Dados de riqueza e abundância de aves foram coletados ao longo de pontos dispostos de forma semialeatória e com pelo menos $300 \mathrm{~m}$ entre si, ao longo de um transecto linear no entorno da Lagoa, de acordo com condições de acessibilidade durante a estação chuvosa e o melhor aproveitamento do tempo em campo (BIBBY et al., 2000). Cada ponto cobriu uma área circular com raio de $300 \mathrm{~m}$ de comprimento. Para cada espécie, considerou-se o valor de abundância do ponto com maior número de aves observado ao longo da amostragem. Em adição, a contagem de bandos foi feita considerando-se classes de cinco indivíduos (WARNOCK et al., 2002). 
Dados de precipitação foram obtidos na estação meteorológica automática da Prefeitura de Quissamã, situada a $4 \mathrm{~km}$ da área de estudo. Considerou-se como amostra o valor total de precipitação para cada mês.

As diferenças de riqueza e abundância entre estações seca e úmida foram testadas por meio do teste- $t$ de Student, considerando-se o nível de significância de 0,05 . Os dados foram transformados em $\log (x+1)$, de acordo com o método de Box-Cox (VERNABLES; RIPLEY, 2002), para cumprir premissas do teste. A influência de diferentes valores de precipitação na abundância de aves foi avaliada com o uso da correlação linear de Spearman. Para isso, as espécies foram agrupadas em seis grupos funcionais (BLONDEL, 2003), de acordo com semelhanças morfo-anatômicas, táticas de forrageio e tipo de item alimentar (GRAAF et al., 1985; MAGALHÃES, 1990; MOTTA-JUNIOR, 1990; SICK, 1997; NTIAMOA-BAIDU et al., 1998). Espécies com Constância (LARRAZÁBAL et al., 2000 ) inferior a $25 \%$ foram desconsideradas para evitar tendências nos resultados. O modelo de predição de abundância de aves aquáticas em função dos valores mensais de precipitação foi selecionado considerandose o que apresentou maior coeficiente de determinação $\left(\mathrm{R}^{2}\right)$ (GUTHERY; BINGHAM, 2007). A eficácia do modelo foi avaliada por meio da correlação linear de Spearman entre dados de abundância previstos pelo modelo e os obtidos no presente estudo, considerandose nível de significância de 0,05 . As análises estatísticas foram realizadas com o software R 3.0.0 (IHAKA; GENTLEMAN, 1996).

\section{Resultados}

No presente estudo foram registradas 50 espécies de aves aquáticas na APA da Lagoa da Ribeira (Tabela 1). Desse total, 18 espécies contaram com registros reprodutivos, concentrados no início da estação seca. As espécies mais abundantes ao longo do período de amostragem foram Dendrocygna viduata (Linnaeus, 1766), Dendrocygna autumnalis (Linnaeus, 1758), Jacana jacana (Linnaeus, 1766) e Gallinula galeata (Lichtenstein,1818), com contagens médias de 123 \pm 171 , $54 \pm 90,53 \pm 46$ e $52 \pm 59$ aves, respectivamente (Figura 2).
A riqueza média foi maior na estação seca $(25 \pm 3$ espécies) do que na estação chuvosa (17 2 espécies) (teste- $\mathrm{t}=-4,85, \mathrm{P}<0,01)$. Da mesma forma, a média de abundância foi maior na seca ( $653 \pm 455$ aves), do que na estação chuvosa ( $143 \pm 90$ aves) (teste- $\mathrm{t}=-3,79, \mathrm{P}$ $=0,003)$. Os meses em que foram registrados maiores valores de abundância foram maio ( 865 aves), junho (1045 aves) e julho (1409 aves). Os meses com menores valores foram novembro ( 88 aves), dezembro ( 85 aves) e janeiro (75 aves) (Figura 3). Observou-se um nítido padrão invertido entre a abundância de aves e valores de precipitação.

TABELA 1: Valores do coeficiente de correlação de Spearman entre a abundância dos grupos funcionais de aves aquáticas e precipitação pluviométrica mensal na Lagoa da Ribeira, Norte Fluminense, sudeste do Brasil. Os asteriscos indicam coeficientes não significativos $(\mathrm{P}>0,05)$.

\begin{tabular}{lcc}
\hline Grupos funcionais & $\boldsymbol{\rho}_{\text {Spearman }}$ & Significância \\
\hline Vasculhadoras & $-0,62$ & $\mathrm{P}=0,03$ \\
Mergulhadoras & $-0,72$ & $\mathrm{P}=0,008$ \\
Pernaltas & $-0,18$ & $*$ \\
Catadoras & $-0,53$ & $\mathrm{P}=0,05$ \\
Limícolas & $-0,13$ & $*$ \\
Pescadoras & $-0,48$ & $*$ \\
\hline
\end{tabular}

O coeficiente de correlação linear de Spearman entre valores mensais de abundância e precipitação foi de -0,77 ( $\mathrm{P}=0,003)$ (Figura 4). Em adição, observouse correlação negativa significativa entre valores de precipitação e a abundância de aves vasculhadoras, mergulhadoras e catadoras (Tabela 1). O modelo de regressão exponencial foi o que melhor representou as flutuações numéricas de aves em função da precipitação na Lagoa da Ribeira $\left(\mathrm{R}^{2}=0,53\right)$. A equação obtida por meio desse modelo foi $\mathrm{Y}=7839 \mathrm{X}^{-0,754}$, onde $\mathrm{Y}$ é número de aves contadas nas condições do presente estudo e $\mathrm{X}$ o valor de precipitação mensal (Figura 4). Em adição, a correlação entre valores previstos pelo modelo e os observados no presente estudo foi $0,76(\mathrm{P}=0,006)$. A transformação em $\log (\mathrm{x}+1)$ permitiu também o ajuste de um modelo linear (Figura 4). 
FIGURA 2: Distribuição da abundância média de aves aquáticas na Lagoa da Ribeira, Quissamã, Norte Fluminense, sudeste do Brasil. São consideradas apenas espécies com número médio de indivíduos superior a 1.

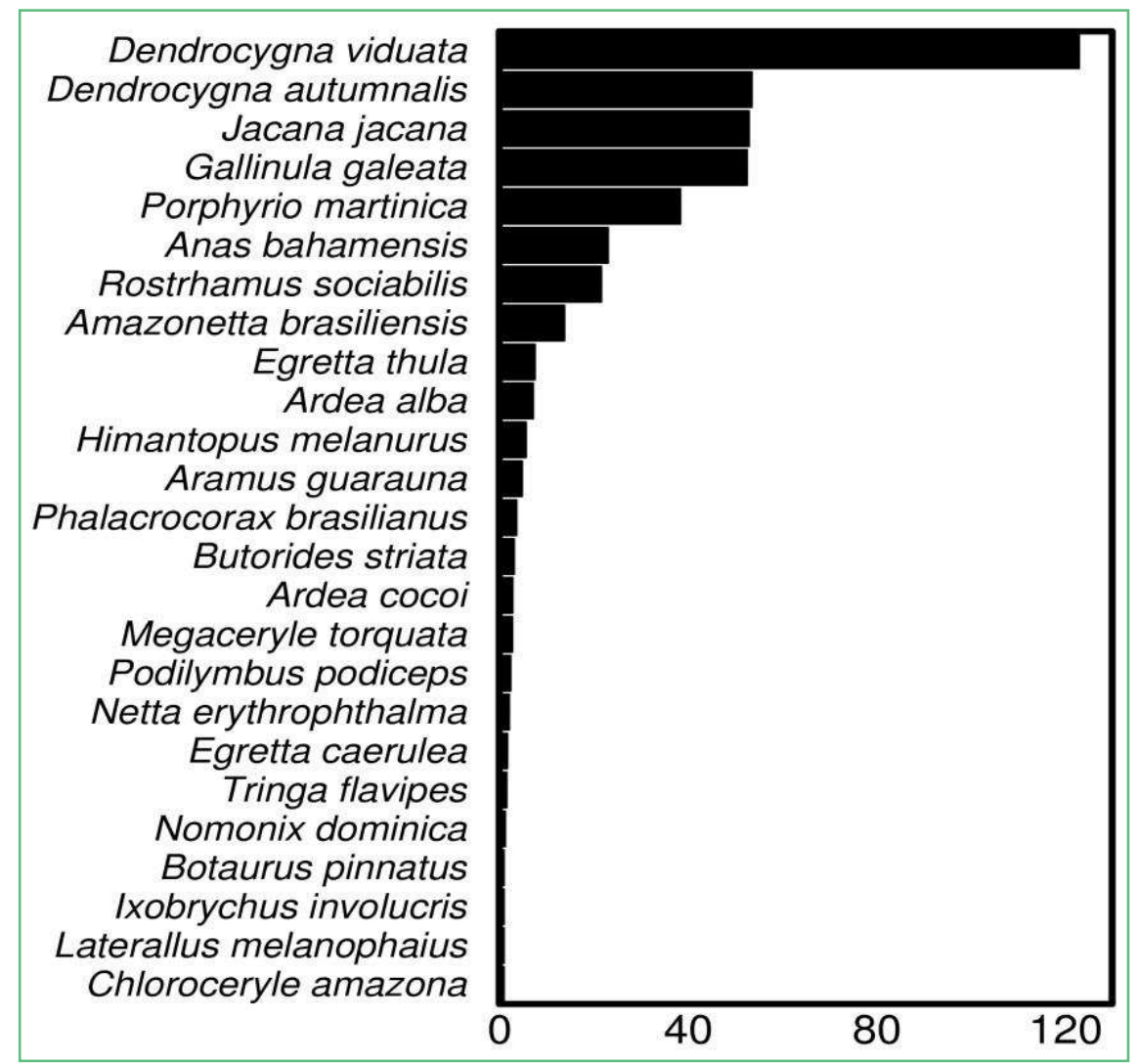

FIGURA 3: Precipitação pluviométrica (linha) e abundância de aves aquáticas (barras) entre agosto de 2008 e julho de 2009 na Lagoa da Ribeira, Norte Fluminense, sudeste do Brasil. A abundância é referente ao ponto com maior número de aves observado ao longo das visitas mensais.

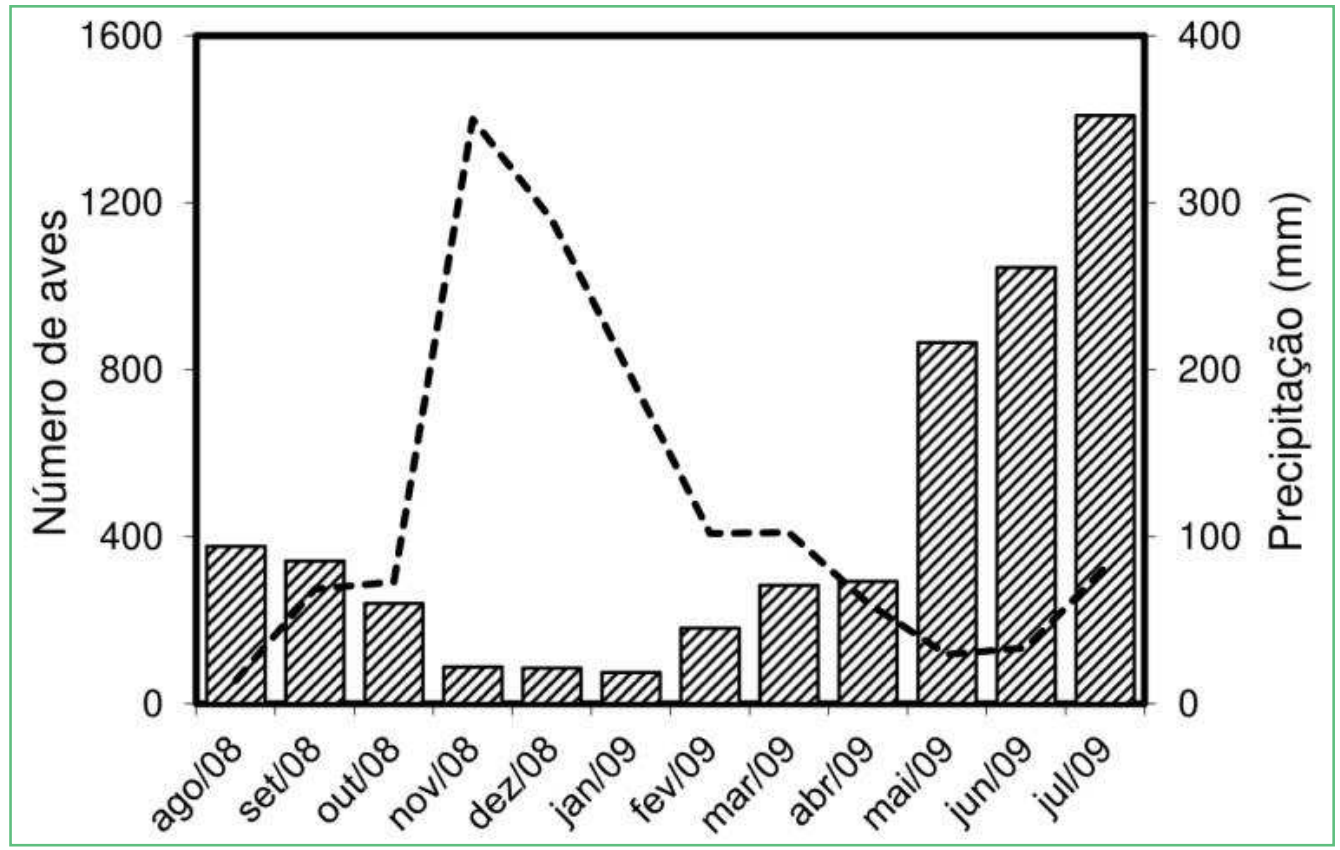


Destaca-se que foram registradas oito espécies aquáticas ameaçadas de extinção em nível estadual (ALVES et al., 2000) (Tabela 2), das quais Dendrocygna bicolor (Vieillot, 1816), Cairina moschata (Linnaeus, 1758) e Ciconia maguari (Gmelin, 1789) estão classificadas na categoria "Vulnerável" e Sarkidiornis sylvicola Ihering \& Ihering, 1907, "Em Perigo", enquanto, Platalea ajaja Vieillot, 1816, Chroicocephalus cirrocephalus (Vieillot, 1818), Himantopus melanurus Vieillot, 1817 e D. autumnalis encontram-se classificadas como "Provavelmente Ameaçadas". Esta última foi registrada em bandos de até 250 aves, um número consideravelmente elevado, tratando-se do estado do Rio de Janeiro.

FIGURA 4: Correlação entre o número de aves e valores de precipitação na Lagoa da Ribeira, Norte Fluminense, sudeste do Brasil. Regressão exponencial em preto e linear em verde, para dados transformados em $\log (\mathrm{x}+1)$.

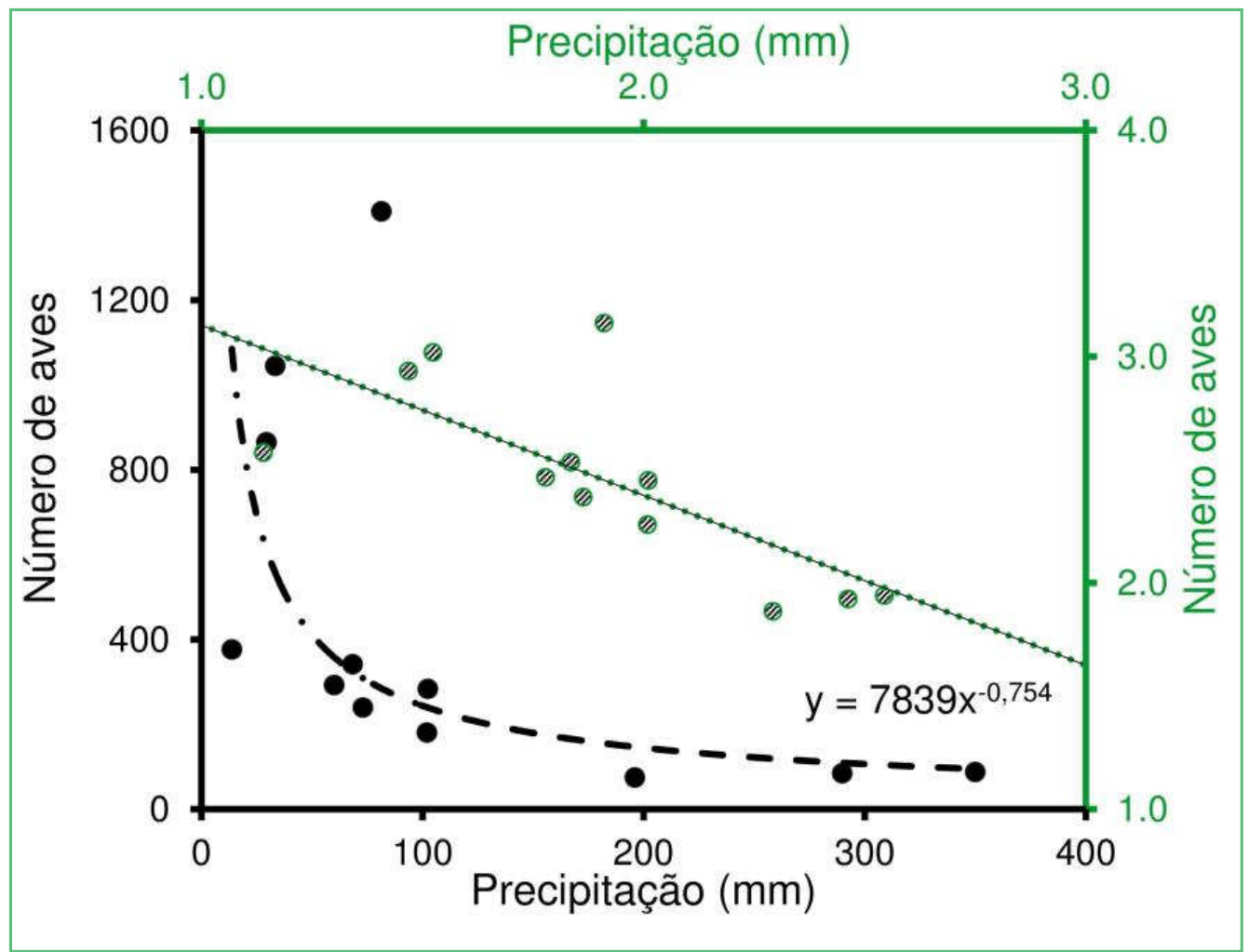


TABELA 2: Grupos funcionais de aves aquáticas registradas na Lagoa da Ribeira, Norte Fluminense, sudeste do Brasil. C - Constância; Estado de conservação: VU - vulnerável, PA - provavelmente ameaçada, EN - em perigo, DD - dados desconhecidos; • - registro reprodutivo; Espécies registradas fora do período de amostragens sistematizadas estão indicadas pelo símbolo $\circ$.

\begin{tabular}{|c|c|c|c|c|}
\hline Grupo Funcional & Espécies & Conservação (Alves 2000) & Repro. & $\mathrm{C}(\%)$ \\
\hline \multirow{10}{*}{ Vasculhadoras } & Dendrocygna viduata & & $\bullet$ & 100 \\
\hline & Dendrocygna autumnalis & PA & - & 91,7 \\
\hline & Amazonetta brasiliensis & & $\bullet$ & 100 \\
\hline & Anas bahamensis & & $\bullet$ & 75 \\
\hline & Netta erythrophthalma & DD & & 41,7 \\
\hline & Nomonix dominica & DD & & 41,7 \\
\hline & Netta peposaca & DD & & 25,0 \\
\hline & Cairina moschata & VU & & $\circ$ \\
\hline & Sarkidiornis sylvicola & EP & & $\circ$ \\
\hline & Dendrocygna bicolor & VU & & 8,3 \\
\hline \multirow{3}{*}{ Mergulhadoras } & Podilymbus podiceps & & - & 66,7 \\
\hline & Tachybaptus dominicus & & & 25 \\
\hline & Phalacrocorax brasilianus & & & 66,7 \\
\hline \multirow{15}{*}{ Pernaltas } & Tigrisoma lineatum & DD & & 25 \\
\hline & Botaurus pinnatus & DD & $\bullet$ & 33,3 \\
\hline & Ixobrychus involucris & & & 33,3 \\
\hline & Butorides striata & & $\bullet$ & 91,7 \\
\hline & Ardea cocoi & & $\bullet$ & 66,7 \\
\hline & Ardea alba & & $\bullet$ & 100 \\
\hline & Egretta thula & & & 100 \\
\hline & Egretta caerulea & & & 58,3 \\
\hline & Ciconia maguari & VU & $\bullet$ & 25 \\
\hline & Aramus guarauna & & $\bullet$ & 91,7 \\
\hline & Ixobrychus exilis & DD & & 8,3 \\
\hline & Syrigma sibilatrix & & & 0 \\
\hline & Nycticorax nycticorax & & & 16,7 \\
\hline & Platalea ajaja & PA & & 8,3 \\
\hline & Pandion haliaetus & & & 8,3 \\
\hline \multirow{10}{*}{ Catadoras } & Rostrhamus sociabilis & & $\bullet$ & 100 \\
\hline & Aramides saracura & & & 25 \\
\hline & Laterallus melanophaius & & & 33,3 \\
\hline & Porzana albicollis & & $\bullet$ & 25 \\
\hline & Gallinula galeata & & $\bullet$ & 100 \\
\hline & Porphyrio martinica & & $\bullet$ & 100 \\
\hline & Jacana jacana & & $\bullet$ & 100 \\
\hline & Gallinula melanops & & & 8,3 \\
\hline & Aramides cajanea & & & 8,3 \\
\hline & Pardirallus nigricans & & & 8,3 \\
\hline \multirow{10}{*}{ Limícolas } & Charadrius collaris & & & 25 \\
\hline & Himantopus melanurus & PA & $\bullet$ & 50 \\
\hline & Tringa solitaria & & & 25 \\
\hline & Tringa flavipes & & & 25 \\
\hline & Phalaropus tricolor & & & 25 \\
\hline & Charadrius semipalmatus & & & $\circ$ \\
\hline & Tringa melanoleuca & & & 16,7 \\
\hline & Calidris melanotos & & & 8,3 \\
\hline & Chroicocephalus cirrocephalus & PA & & 8,3 \\
\hline & Larus dominicanus & & & $\circ$ \\
\hline \multirow{2}{*}{ Pescadoras } & Megaceryle torquata & & $\bullet$ & 91,7 \\
\hline & Chloroceryle amazona & & & 33,3 \\
\hline
\end{tabular}




\section{Discussão}

Áreas úmidas continentais se distribuem como mosaicos de ambientes, portanto diferem entre si quanto à composição e abundância das aves aquáticas (HAIG et al., 1998; ACCORDI; HARTZ, 2006). Nesse contexto, cada espécie seleciona a área com características bióticas e abióticas mais adequadas à sua sobrevivência e reprodução (SINCLAIR, 1989; ELPHICK; ORING, 1998; WELLER, 2003). Isso faz com que regiões com características mais apropriadas, em geral, suportem maior abundância de aves aquáticas (MA et al., 2010). Os resultados obtidos no presente trabalho confirmam que os regimes de precipitação são determinantes na variação temporal de aves aquáticas da Lagoa da Ribeira.

Em geral, trabalhos conduzidos em diferentes latitudes do país têm detectado maior abundância de aves aquáticas durante a estação seca, quando se considera espécies residentes, ou seja, que se reproduzem na região (RODRIGUES; MICHELIN, 2005; ACCORDI; HARTZ, 2006; BARBIERI, 2007; SOARES; RODRIGUES, 2009). Durante o período seco em áreas úmidas, existe um padrão de maior disponibilidade de alimento para espécies que forrageiam principalmente vegetais presentes em águas rasas, como D. viduata e D. autumnalis (SICK, 1997; NTIAMOABAIDU et al., 1998). Como estas espécies contribuíram em grande parte para o incremento numérico de aves no período seco, a maior disponibilidade de alimento durante o período seco na Lagoa da Ribeira pode ser uma explicação para o aumento do número de aves neste período. Além disso, a maior abundância de aves durante a estação seca também pode ser atribuída ao recrutamento de jovens após o período reprodutivo das espécies mais abundantes na Lagoa da Ribeira, conforme observado por Alves e Pereira (1998) na Lagoa Rodrigo de Freitas/RJ.

O modelo de predição exponencial apresentou maior sentido biológico quando comparado a outros modelos, incluindo-se o linear. Este último retorna valores de abundância negativos (irreais) sob condições elevadas de precipitação. De acordo com o modelo exponencial obtido, espera-se considerável declínio na abundância de aves aquáticas em meses com precipitação superior a $100 \mathrm{~mm}$. Isso muito provavelmente devido à menor disponibilidade de profundidades d'água adequadas a alimentação de diferentes espécies, que dificulta o acesso à presas e reduz áreas para pouso e descanso (PARACUELLOS; TELLERÍA, 2004; KOBER; BAIRLEIN, 2009; NORES, 2011). Esse fenômeno foi claramente observado durante as amostragens em campo. O elevado coeficiente de correlação de Spearman entre valores previstos pelo modelo e os obtidos no presente estudo indica grande eficácia deste modelo simples, baseado em apenas uma variável ambiental: precipitação pluviométrica mensal.

A maior abundância de aves vasculhadoras durante o período seco concorda com as observações de Tavares e Siciliano (2013a). Nesse período, plantas e sementes que servem de alimento (BOVE; PAZ, 2009) estão mais acessíveis, devido à predominância de lâminas d'água com baixa profundidade (KOBER; BAIRLEIN, 2009; LUNARDI et al., 2012). Da mesma forma, a maior abundância de limícolas na seca está associada principalmente ao acesso de presas bentônicas. Tringa flavipes e Tringa solitaria, por exemplo, dependem de águas com profundidades de $0-15 \mathrm{~cm}$ para alcançar as presas (MA et al., 2010). A mesma situação foi reportada em um estudo conduzido em alagados nos Lençois Maranhenses, norte do Brasil (SOARES; RODRIGUES, 2009). No entanto, trabalhos conduzidos em áreas úmidas de diferentes regiões do Brasil e do mundo têm obtido valores de abundância e biomassa de invertebrados opostos para mesmos períodos climáticos (e. g. REISS, 1977; CENZANO; WÜRDIG, 2006; ERSAN et al., 2009). Em algumas regiões, como no Rio Grande do Sul, sul do Brasil, a densidade de macroinvertebrados tem sido muito maior na estação úmida do que na seca. Isso reforça a importância de estudos que levem em consideração as variações específicas de cada região. Nesse contexto, estudos a cerca da dieta das aves aquáticas do Norte Fluminense permitiriam o melhor entendimento das relações espécie-hábitat e o manejo das áreas úmidas degradadas na região.

A menor abundância de aves catadoras durante o período úmido pode ser explicada por múltiplos fatores ligados aos diferentes grupos taxonômicos pertencentes a esse grupo funcional, que une frangos 
d'água, saracuras e o gavião-caramujeiro (R. sociabilis). É provável que os frangos d'água se desloquem para lagoas costeiras adjacentes a linha de praia no interior do Parque Nacional da Restinga de Jurubatiba, onde ocorrem em maior abundância durante a estação úmida (TAVARES; SICILIANO, 2013b). Algumas dessas lagoas possuem grande disponibilidade de vegetais adequados a sua dieta (TELINO-JÚNIOR et al., 2003; NUNES; PITARELLI, 2005; BOVE; PAZ, 2009), aparentemente em maior abundância na estação úmida. Já a maior abundância de Rosthramus sociabilis em meses com baixa precipitação parece estar associada à maior abundância de caramujos do gênero Pomacea (Perry, 1810), que em determinadas regiões podem ocorrer na seca (ABÍLIO et al., 2006; SOUZA; ABÍLIO, 2006). No entanto, em períodos chuvosos há maior disponibilidade de caramujos associados à vegetação (SICK, 1997). Na Estação Ecológica do Taim, por exemplo, foi relatada maior abundância de $R$. sociabilis durante o verão (MÄHLER, 1996). Mais fatores que influenciem os prováveis deslocamentos da espécie devem ser investigados, além da disponibilidade de alimento. Quanto às saracuras (Rallidae), embora exista um padrão de maior abundância destas aves em áreas de vegetação densa (e.g. Typha domingensis), mais disponíveis durante o período chuvoso, a variação de abundância dessas aves contribuiu pouco para a correlação negativa entre a abundância de aves catadoras e os valores de precipitação pluviométrica.

Um estudo no complexo de lagoas costeiras em Quissamã, Norte Fluminense, detectou maior densidade de peixes durante a estação seca (MACEDO-SOARES et al., 2010). O aprisionamento de peixes em elevadas densidades e em corpos d'água de área reduzida facilita a detecção e captura por aves pescadoras, pernaltas e algumas limícolas (HERRING; GAWLIK, 2011). Espécies cujo estado de conservação é delicado, como C. maguari e C. cirrocephalus (TABELA 1) tiveram ocorrência restrita à estação seca, provavelmente devido à melhor condição de forrageio, conforme observado por Tavares et al. (2012) em outras lagoas costeiras do Norte Fluminense.

Outro possível fator que contribui para o declínio na abundância de aves durante a estação chuvosa é a maior suscetibilidade à predação. Nesse período, potenciais predadores como Caiman latirostris (Daudin, 1802), Boa constrictor (Linnaeus, 1758) e Lontra longicaudis (Olfers, 1818) ampliam sua área de distribuição na lagoa, o que pode resultar no afugentamento de aves (QUINTELA et al., 2008; BORTEIRO et al., 2009; PIZZATO et al., 2009). Rheingantz et al. (2011), por exemplo, constataram a presença de aves na dieta de L. longicaudis apenas durante a estação chuvosa na planície costeira de Angra dos Reis, sul do estado do Rio de Janeiro.

Alguns autores mencionam que o tamanho dos corpos d'água é um dos principais fatores de predição da abundância de aves aquáticas, ou seja, quanto maior a área do espelho d'água, maior será abundância (PARACUELLOS; TELLERÍA, 2004). Contudo, o aumento de área implica, em determinadas situações, no aumento de profundidade d'água e alteração de parâmetros físico-químicos (ESTEVES, 1998; HOLM; CLAUSEN, 2006). Em algumas situações isso poderia ser benéfico para aves mergulhadoras, embora não tenha sido no presente estudo. Por outro lado, certamente seria muito prejudicial para espécies limícolas, pernaltas e vasculhadoras, devido às suas limitações morfo-anatômicas para pouso e forrageio. Com área relativamente pequena, a Lagoa da Ribeira é um bom exemplo dessa circunstância.

Há poucos estudos focados na ecologia de aves aquáticas no estado do Rio de Janeiro, no entanto nossos dados podem ser comparados com os obtidos por Alves e Pereira (1998), na Lagoa Rodrigo de Freitas, situada na costa central do estado do Rio de Janeiro. Nessa lagoa, a riqueza de aves aquáticas não diferiu significativamente ao longo do ano, em contraste com a diferença observada entre os períodos seco e chuvoso na Lagoa da Ribeira. Nessa última, a maior riqueza de aves aquáticas na estação seca ocorreu devido à ocorrência restrita de espécies incomuns no estado do Rio de Janeiro (GAGLIARDI, 2011) que, em geral, dependem de águas rasas para alimentação, como $P$. ajaja, Phalaropus tricolor (Vieillot, 1819), Calidris melanotus (Vieillot, 1819), S. sylvicola e Netta erythrophthalma (Wied, 1832) (SICK, 1997; TAFT et al., 2002). Na estação úmida houve também baixo influxo de migratórias 
neárticas (apenas 6 espécies) (MESTRE et al., 2010). Essas espécies parecem utilizar principalmente a cadeia de lagoas adjacentes à linha de praia no município, as quais apresentam maior disponibilidade de solos lodosos para alimentação (TAVARES et al., 2012; TAVARES; SICILIANO, 2013b).

No contexto de mudanças climáticas, os modelos atuais preveem que o sudeste do Brasil passará por eventos extremos de chuvas, com maior frequência durante a segunda metade do século XXI (MARENGO et al., 2009). Diante dos resultados obtidos no presente estudo, estes eventos poderão afetar drasticamente a estrutura da assembleia de aves aquáticas na Lagoa da Ribeira e entorno. Essa possibilidade justifica que áreas alagadas com importância para aves aquáticas, sejam monitoradas continuamente e em longo prazo, para detecção de tendências populacionais.

É relevante mencionar que a APA da Lagoa da Ribeira já sofreu considerável perda de áreas naturais ao longo de sua história (mapas disponíveis no centro cultural do município de Quissamã). Esse fato pode ser reforçado pelas estimativas de área da Lagoa Feia, a qual teve sua extensão reduzida em aproximadamente em 50\% ao longo do último século (SOFFIATI, 1998). Durante uma visita à Lagoa da Ribeira, no ano de 2012, observou-se notória substituição de suas áreas naturais por pastos, plantios de espécies exóticas e residências ilegais. Além disso, novos canais de drenagem foram implantados.

As flutuações de abundância de aves aquáticas na APA da Lagoa da Ribeira são diretamente influenciadas pelas variações de precipitação. Porém, não há dúvidas de que outras variáveis também influenciam as flutuações de abundância de aves na região. Portanto, o modelo de predição obtido pode ter sua acurácia aumentada com a incorporação de outras variáveis físico-químicas e biológicas.

\section{Agradecimentos}

Os autores são gratos ao Programa de Pósgraduação em Ecologia e Recursos Naturais da Universidade Estadual do Norte Fluminense (UENF) e à Prefeitura de Quissamã. D.C. Tavares recebe auxílio para pesquisa da Coordenação de Aperfeiçoamento de Pessoal de Nível Superior-CAPES. Os custos para publicação foram financiados pelo Programa de Apoio à Pós-Graduação (PROAP).

\section{Referências}

ABÍLIO, F. J. P.; FONSECA-GESSNER, A. A.; LEITE, R. L.; RUFFO, T. L. M. Gastrópodes e outros invertebrados do sedimento e associados à macrófita Eichhornia crassipes de um açude hipertrófico do semi-árido paraibano. Revista de Biologia e Ciências da Terra, Campina Grande, v. 1, p. 165-178, 2006.

ACCORDI, I. A.; HARTZ, S. M. Distribuição espacial e sazonal da avifauna em uma área úmida costeira do sul do Brasil. Revista Brasileira de Ornitologia, São Leopoldo, v. 2, n. 14, p. 117-135, 2006.

ALVES, M. A. S.; PACHECO, J. F.; GONZAGA, L. A. P.; CAVALCANTI, R. B.; RAPOSO, M. A.; YAMASHITA, C.; MACIEL, N. C.; CASTANHEIRA, M. Aves. In: BERGALLO, H. G.; ROCHA, C. F. D.; ALVES, M. A. S.; SLUYS, M. V. (Ed.). A fauna ameaçada de extinção do estado do Rio de Janeiro. Rio de Janeiro: Ed. UERJ, 2000. p. 117-124.

ALVES, M. A. S.; PEREIRA, E. F. Richness, abundance and seasonality of bird species in a lagoon of an urban area (Lagoa Rodrigo de Freitas) of Rio de Janeiro, Brazil. Ararajuba, Londrina, v. 6, n. 2, p. 110-116, 1998.

ARAÚJO, D. S. D.; SCARANO, F. R.; SÁ, C. F. C.; KURTZ, B. C.; ZALUAR, H. L. T.; MONTEZUMA, R. C. M.; OLIVEIRA, R. C. Comunidades vegetais do Parque Nacional da Restinga de Jurubatiba. In: ESTEVES, F. A. (Ed.). Ecologia de lagoas costeiras do Parque Nacional da Restinga de Jurubatiba e do município de Macaé, RJ. Macaé: Universidade Federal do Rio de Janeiro, 1998. p. 39-62.

BARBIERI, E. Seasonal abundance of shorebirds at Aracaju, Sergipe, Brazil. Wader Study Group Bulletin, Devon, v. 113, p. 40-46, 2007.

BIBBY, C.; MARTIN, J.; MARSDEN, S. Expedition field techniques: bird surveys. Cambridge: BirdLife International, 2000. $137 \mathrm{p}$.

BLANCO, D. E. Los humedales como hábitat de aves acúaticas. In: MALVÁREZ, A. I. (Ed.). Tópicos sobre humedales subtropicales y templados de Sudamérica. Montevideo: Orcyt-Unesco, 1999. p. 208-217.

BLONDEL, J. Guilds or functional groups: does it matter? Oikos, Copenhagen, v. 100, p. 223-231, 2003.

BOLDUC, F.; AFTON, A. D. Relationships between wintering waterbirds and invertebrates, sediments and hydrology of coastal marsh ponds. Waterbirds, Waco, v. 27, p. 333-341, 2004.

BORTEIRO, C.; GUTIÉRREZ, F.; TEDROS, M.; KOLENC, F. Food habits of the Broad-snouted Caiman (Caiman latirostris: Crododylia, Alligatoridae) in northwestern Uruguay. Studies on Neotropical Fauna and Environmental, London, v. 44, n. 1, p. 31-36, 2009. 
BOVE, C. P.; PAZ, J. Guia de campo das plantas aquáticas do Parque Nacional da Restinga de Jurubatiba. Rio de Janeiro: Museu Nacional, 2009. 176 p.

CENZANO, C. S. S.; WÜRDIG, N. L. Spatial and temporal variations of the benthic macrofauna in different habitats of a lagoon of the northern coastal system of Rio Grande do Sul State, Brazil. Acta Limnologica Brasiliensia, Botucatu, v. 18, n. 2, p. 153-163, 2006.

DIMALEXIS, A.; PYROVETSI, M.; SGARDELIS, S. Effect of water level fluctuations on wading bird foraging habitat use at an irrigation reservoir, Lake Kerkini. Colonial Waterbirds, Waco, v. 20, p. 244-252, 1997.

ELPHICK, C. S.; ORING, L. W. Winter management of Californian rice fields for waterbirds. Journal of Applied Ecology, London, v. 35, p. 95-108, 1998.

ERSAN, E.; ALTINDAG, A.; AHISKA, S.; ALAS, A. Zoobenthic fauna and seasonal changes of mamasin dam lake (Central part of Turkey). African Journal of Biotechnology, Cape Town, v. 8, n. 18, p. 4702-4707, 2009.

ESTEVES, F. A. Fundamentos de Limnologia. 2. ed. Rio de Janeiro: Interciência, 1998. 226 p.

GAGLIARDI, R. Lista de aves do estado do Rio de Janeiro. 2011. Disponível em: <http://www.ceo.org.br/listas_de_aves/RJGagliardi.pdf $>$. Acesso em: 10 fev. 2012.

GRAAF, R. M.; TILGHMAN, N. G.; ANDERSON, S. H. Foraging guilds of North American Birds. Environmental Management, New York, v. 9, n. 6, p. 493-536, 1985.

GUTHERY, F. S.; BINGHAM, R. L. A Primer on interpreting regression models. The Journal of Wildlife Management, Bathesda, v. 71, n. 3, p. 684-692, 2007.

HAIG, S. M.; WEHLMAN, D. W.; ORING, L. W. Avian movements and wetland connectivity in landscape conservation. Conservation Biology, San Francisco, v. 12, p. 749-758, 1998.

HERRING, H. K.; GAWLIK, D. Resource selection functions for wood stork foraging habitat in the southern Everglades. Waterbirds, Waco, v. 34, n. 2, p. 133-142, 2011.

HOLLANDA-CARVALHO, P.; SÁNCHEZ-BOTERO, J. I.; PELLEGRINI-CARAMASCHI, E.; BOZELLI, R. L. Temporal variation of fish community richness in coastal lagoons of the Restinga de Jurubatiba National Park, Rio de Janeiro, Brazil. Acta Limnologica Brasiliensia, São Carlos, v. 15, n. 3, p. 31-40, 2003.

HOLM, T.; CLAUSEN, P. Effects of water level management on autumn staging waterbird and macrophyte diversity in three Danish coastal lagoons. Biodiversity and Conservation, New York, v. 15, n. 14, p. 4399-4423, 2006.

IHAKA, R.; GENTLEMAN, R. R: a language for data analysis and graphics. Journal of Computational and Graphical Statistics, Alexandria, v. 5, p. 299-314, 1996.

ISOLA, C. R.; COLWELL, M. A.; TAFT, O. W.; SAFRAN, R. J. Interspecific differences in habitat use of shorebirds and waterfowl foraging in managed wetlands of California's San Joaquin Valley. Waterbirds, Waco, v. 23, n. 2, p. 196-203, 2000.

KJERFVE, B. Comparative oceanography of coastal lagoons. In: WOLFE, D. A. (Ed.). Estuarine variability. New York: Academic Press, 1986. p. 63-81.
KOBER, K.; BAIRLEIN, F. Habitat choice and niche characteristics under poor food conditions. A study on migratory nearctic shorebirds in the intertidal flats of Brazil. Netherlands Ornithologists Union, Ewijk, v. 97, n. 1, p. 31-42, 2009.

LAMEGO, A. R. O homem e o brejo. Rio de Janeiro: Conselho Nacional de Geografia, 1945. 204 p.

LARRAZÁBAL, M. E.; AZEVEDO-JÚNIOR, S. M.; PENA, O. Monitoramento de aves limícolas na salina Diamante Branco, Galinhos, Rio Grande do Norte, Brasil. Revista Brasileira de Ornitologia, São Leopoldo, v. 19, n. 4, p. 1081-1089, 2000.

LUNARDI, V. O.; MACEDO, R. H.; GRANADEIRO, J. P.; PALMEIRIM, J. M. Migratory flows and foraging habitat selection by shorebirds along the northeastern coast of Brazil: the case of Baía de Todos os Santos. Estuarine, Coastal and Shelf Science, New York, v. 96, p. 179-187, 2012.

MA, Z.; CAI, Y.; LI, B.; CHEN, J. Managing wetland habitats for waterbirds: an international perspective. Wetlands, Gdansk, v. 30, n. 1, p. 15-27, 2010.

MACEDO-SOARES, P. H. M.; PETRY, A. C.; FARJALLA, V. F.; CAMARASCHI, E. P. Hydrological connectivity in a coastal inland systems: lessons from a Neotropical fish metacommunity. Ecology of Freshwater Fish, Malden, v. 19, n. 1, p. 7-18, 2010.

MAGALHÃES, C. A. Hábitos alimentares e estratégia de forrageamento de Rostrhamus sociabilis no Pantanal de Mato Grosso, Brasil. Ararajuba, Londrina, v. 1, p. 95-98, 1990.

MÄHLER, J. K. F. Lista comentada das espécies de aves da Estação Ecológica do Taim, Rio Grande do Sul. Acta Biologica Leopoldensia, São Leopoldo, v. 18, p. 69-103, 1996.

MARENGO, J. A.; JONES, R.; ALVES, L. M.; VALVERDE, M. C. Future change of temperature and precipitation extremes in South America as derived from the PRECIS regional climate modeling system. International Journal of Climatology, Malden v. 29, n. 15, p. 2241-2255, 2009.

MAROTTA, H.; DUARTE, C. M.; PINHO, L.; ENRICH-PRAST, A. Rainfall leads to increased $p \mathrm{CO}_{2}$ in Brazilian coastal lakes. Biogeosciences, Orono, v. 7, p. 1607-1614, 2010.

MESTRE, L. A. M.; ROOS, A. L.; NUNES, M. F. Análise das recuperações no Brasil de aves anilhadas no exterior entre 1927 e 2006. Ornithologia, Palmas, v. 4, n. 1, p. 15-35, 2010.

MILLAN-NUÑEZ, R.; ALVAREZ-BORREGO, S.; NELSON, D. M. Effects of physical phenomena on the distribution of nutrients and phytoplankton productivity in a coastal lagoon. Estuarine, Coastal and Shelf Science, New York, v. 15, n. 3, p. 317-335, 1982. MOTTA-JUNIOR, J. C. Estrutura trófica e composição das avifaunas de três hábitats terrestres na região central do estado de São Paulo. Ararajuba, Londrina, v. 1, p. 65-71, 1990.

NESSIMIAN, J. L. Composição da fauna de invertebrados bentônicos em um brejo entre dunas no litoral do estado do Rio de Janeiro, Brasil. Acta Limnologica Brasiliensia, Botucatu, v. 7, p. 41-59, 1995.

NTIAMOA-BAIDU, Y.; PIERSMA, T.; WIERSMA, P.; POOT, M.; BATTLEY, P.; GORDON, C. Water depth selection, daily feeding routines and diets of waterbirds in coastal lagoons in Ghana. Ibis, Peterborough, v. 140, p. 89-103, 1998.

NORES, M. Long-term waterbird fluctuations in Mar Chiquita Lake, Central Argentina. Waterbirds, Waco, v. 34, n. 3, p. 381388, 2011. 
NUNES, A. P.; PITARELLI, A. Comportamento da jaçana (Jacana jacana Linnaeus, 1766) (Charadriiformes, Jacanidae) em uma lagoa urbana no município de Três Lagoas, Mato Grosso do Sul, Brasil. Atualidades Ornitológicas, Ivaiporã, v. 126, p. 1-17, 2005. PARACUELLOS, M.; TELLERÍA, J. L. Factors affecting the distribution of a waterbird community: the role of habitat configuration and bird abundance. Waterbirds, Waco, v. 27, n. 4, p. 446-453, 2004

PIZZATO, L.; MARQUES, O. A. V.; FACURE, K. Food habits of Brazilian boid snakes: overview and new data, with special reference to Corallus hortulanus. Amphibia-Reptilia, Leiden, v. 30, p. 533-544, 2009.

QUINTElA, F. M.; PORCIUNCULA, R. A.; COLARES, E. P. Dieta de Lontra longicaudis (Olfers) (Carnivora, Mustelidae) em um arroio costeiro da região sul do Estado do Rio Grande do Sul, Brasil. Neotropical Biology and Conservation, São Leopoldo, v. 3, n. 3, p. 119-125, 2008.

RAMSAR CONVENTION. Ramsar strategic plan 2009-1015. 2009. Disponível em <http://www.ramsar.org/pdf/key_strat plan_2009_e.pdf $>$. Acesso em: 10 fevereiro 2012.

REISS, F. Qualitative and quantitative investigations on the macrobenthic fauna of Central Amazon lakes. Amazoniana, Plön, v. 4, n. 2, p. 203-235, 1977.

RHEINGANTZ, M. L.; WALDEMARIN, H. F.; RODRIGUES, L.; MOULTON, T. P. Seasonal and spatial differences in feeding habits of the Neotropical otter Lontra longicaudis (Carnivora: Mustelidae) in a coastal catchment of southeastern Brazil. Zoologia, Curitiba, v. 28, n. 1, p. 37-44, 2011.

RODRIGUES, M.; MICHELIN, V. B. Riqueza e diversidade de aves aquáticas de uma lagoa natural no sudeste do Brasil. Revista Brasileira de Zoologia, Curitiba, v. 22, n. 4, p. 928-935, 2005.

RUTSCHKE, E. Waterfowl as bio-indicators. International council for bird preservation Technical Publication $n$ 6. Cambridge: Council for Bird Preservation, 1987. 167-172 p.

SICK, H. Ornitologia brasileira. Rio de Janeiro: Nova Fronteira, 1997. 912 p.

SINCLAIR, A. R. E. The regulation of animal populations. In: CHERRET, J. (Ed.). Ecological concepts. Oxford: Blackwell Scientific Publications, 1989. p. 197-241.

SOARES, R. K. P.; RODRIGUES, A. A. F. Distribuição espacial e temporal da avifauna aquática no Lago de Santo Amaro, Parque Nacional dos Lençóis Maranhenses, Maranhão, Brasil. Revista Brasileira de Ornitologia, São Leopoldo, v. 17, n. 3-4, p. 173-182, 2009.
SOFFIATI, A. N. A agonia das lagoas do Norte Fluminense. Ciência e Cultura, São Paulo, v. 37, p. 1628-1638, 1985.

SOUZA, A. H. F. F.; ABÍLIO, F. J. P. Zoobentos de duas lagoas intermitentes da caatinga paraibana e as influências do ciclo hidrológico. Revista de Biologia e Ciências da Terra, Campina Grande, n. 1, p. 146-164, 2006.

TAFT, O. W.; COLWELL, M. A.; ISOLA, C. R.; SAFRAN, R. J. Waterbird responses to experimental drawdown: implications for the multispecies management of wetland mosaics. Journal of Applied Ecology, London v. 39, n. 6, p. 987-1001, 2002.

TAVARES, D. C.; MOURA, J. F.; SICILIANO, S. As aves das áreas úmidas. Ciência Hoje, Rio de Janeiro, v. 50, n. 299, p. 42-47, 2012.

TAVARES, D. C.; SICILIANO, S. Registro documentado de cabeça-seca Mycteria americana no Norte Fluminense após 18 anos sem relatos de ocorrência. Atualidades Ornitológicas, Ivaiporã, v. 172, p. 18-21, 2013a.

TAVARES, D. C.; SICILIANO, S. An inventory of wetland nonpasserine birds along a southeastern Brazilian coastal area. Journal of Threatened Taxa, Nova Delhi, v. 5, n. 11, p. 4586-4597, 2013 b. TELINO-JÚNIOR, W. R.; AZEVEDO-JÚNIOR, S. M.; LYRANEVES, R. M. Biologia e censo e Porphyrula martinica, Gallinula chloropus e Jacana jacana em Dois Irmãos, Pernambuco, Brasil. Lundiana, Belo Horizonte v. 4, n. 1, p. 43-49, 2003.

VERNABLES, W. N.; RIPLEY, B. D. Modern applied statistics with S. New York: Springer, 2002. 495 p.

VOOREN, C. M.; BRUSQUE, L. F. As aves do ambiente costeiro do Brasil. 1999. Disponível em: <http://vivamarajo.org.br/files/ biotico/As-aves-do-ambiente-costeiro-do-Brasil-biodiversidade-econs.pdf $>$. Acesso em: 4 jan. 2010

WARNOCK, N.; PAGE, G. W.; RUHLEN, T. D.; NUR, N.; TAKEKAWA, J. Y.; HANSON, J. T. Management and conservation of San Francisco Bay Salt Ponds: effects of pond salinity, area, tide, and season on pacific flyway waterbirds. Waterbirds, Waco, v. 25 (Special Publication 2), p. 79-92, 2002.

WELLER, M. W. Wetland birds: habitat resources and conservation implications. Cambridge: Cambridge University Press, 2003. 269 p.

WORLD RESOURCES INSTITUTE. Ecosystems and human well-being: biodiversity synthesis. Washington: World Resources Institute, 2005. 86 p. 